\title{
Evaluation of the feasibility of a novel distance adaptable Steady- State Visual Evoked Potential based Brain-Computer Interface *
}

\author{
Chi-Hsu Wu, Heba Lakany
}

Abstract- Steady-state visual evoked potential (SSVEP) based brain-computer interface (BCI) has attracted great attention in BCI research due to its advantages over the other electroencephalography (EEG) based BCI paradigms, such as high speed, high signal to noise ratio, high accuracy, commands scalability and minimal user training time. Several studies have demonstrated that SSVEP BCI can provide a reliable channel to the users to communicate and control an external device. While most SSVEP based BCI studies focus on encoding the visual stimuli, enhancing the signal detection and improving the classification accuracy, there is a need to bridge the gap between BCI "bench" research and real world application. This study proposes a novel distance adaptable SSVEP based BCI paradigm which allows its users to operate the system in a range of viewing distances between the user and the visual stimulator. Unlike conventional SSVEP BCI where users can only operate the system at a fixed distance in front of the visual stimulator, users can operate the proposed BCI at a range of viewing distances. 10 healthy subjects participated in the experiment to evaluate the feasibility of the proposed SSVEP BCI. The visual stimulator was presented to the subjects at 4 viewing distances, $60 \mathrm{~cm}, 150 \mathrm{~cm}, 250 \mathrm{~cm}$ and $350 \mathrm{~cm}$. The mean $\rightarrow$ classification accuracy across the subjects and the viewing distances is over $\mathbf{7 5 \%}$. The results demonstrate the feasibility of a distance adaptable SSVEP based BCI.

\section{INTRODUCTION}

Brain-Computer Interface (BCI) translates users' intents into commands by detecting the brain activities without relying on the brain normal output pathway $[1,2]$. Several electroencephalography (EEG) based BCI paradigms have demonstrated that $\mathrm{BCI}$ can provide a reliable alternative channel for healthy people as well as patients with neuromuscular disorder to communicate and control an external device. Among these EEG based BCI paradigms, SSVEP based $\mathrm{BCI}$ has drawn relatively considerable attention in BCI studies recently due to its high information transfer rate (ITR), high signal to noise ratio (SNR), high classification accuracy, ease of increasing the commands and minimal user training time [3, 4].

The mean classification accuracies and ITR reported in Bremen-BCI speller based on SSVEP were over $95 \%$ and over $60 \mathrm{bits} / \mathrm{min}$ [5]. The highest ITR from one subject in their study was up to $124 \mathrm{bits} / \mathrm{min}$. An environment controller utilizing 48 LEDs achieved ITR of $68 \mathrm{bits} / \mathrm{min}$ [6]. Recently, a mental speller based on SSVEP allowed one

* This study is funded by Engineering and Physical Science Research Council (EPSRC).

Both authors are with Biomedical Engineering Department of University of Strathclyde, Glasgow, Scotland, UK.

e-mail \{chihsu.wu, heba.lakany\}@strath.ac.uk letter to be spelled in one selection facilitated by a QWERT keyboard which contained 30 LEDs representing 26 letters and 4 symbols [7]. These studies have shown the high ITR and classification accuracies in SSVEP based BCIs. They also demonstrated that the superior performance of SSVEP BCI can be achieved by one single selection task or a sequential selection tasks.

There were a few SSVEP based BCI studies which attempted to improve SSVEP detection rates and reduce the calibration phase which is normally used to decide the best parameters for individual subject. Lin et al. (2006) proposed a SSVEP detection method based on canonical correlation analysis (CCA). Their proposed CCA SSVEP detection method found CCA correlation coefficients between EEG signals and the reference signal corresponding to one stimulus. The corresponding stimulus reference signal producing the largest CCA correlation coefficient was regarded as the attended target. Friman et al. (2007) proposed a SSVEP detection method which combined the multiple electrodes signals into a channel signal to enhance SSVEP response and cancel the noise. Both methods outperformed the conventional power spectral density analysis $[8,9]$.

Meanwhile, a great effort has been made to overcome the limitation of the number of the frequencies available to encode the visual stimuli. Jia et al. (2011) used the frequency and the phase to encode SSVEP visual stimuli. More visual stimuli can be created by fewer frequencies. In their study, 15 visual stimuli were made by three distinct frequencies. 4 stimulating frequencies can present 6 to 10 visual stimuli by using dual frequencies for one stimulus $[11,12]$. Multiple frequencies sequential coding (MFSC) presented one stimulus by multiple frequencies in a sequential order. The maximum number of stimuli using MFSC depended on the number of the coding epochs in one sequence and the number of the stimulating frequencies [13]. For example, if the coding epoch is 2 and the number of the frequencies is 3 , the maximum number of stimuli is up to 9 .

Currently, a few commercial EEG headsets available, e.g. Emotiv, which provide user friendly EEG acquisition, have been evaluated and used in BCI studies [14]. The aforementioned studies have offered a solid theory background in the implantation of SSVEP based BCI, from the fundamental visual stimuli to SSVEP detection. Therefore, there is a need to bridge the gap of BCI studies to real world application.

This study proposes a novel SSVEP based BCI which can adapt the change of the viewing distance between the users and the visual stimuli and allows its users to operate the $\mathrm{BCI}$ system at the distance within the range. In conventional 
SSVEP based BCI, the users have to be in front of the visual stimuli in order to elicit SSVEP response to operate BCI. The proposed new paradigm can improve the usability and flexibility. It is hoped that the proposed paradigm can broaden the potential target $\mathrm{BCI}$ users and the applications.

This paper is organized as follows: In Section II, the experimental setup, protocol, and data analysis are presented. Section III presents the analyzed results. Finally, a general discussion is presented in Section IV.

\section{MethodS AND MATERIALS}

\section{A. Subjects}

10 healthy subjects $(8$ males, 2 females, mean age $=30.3$, $\mathrm{SD}=7.9$ ) with normal or corrected to normal vision participated in the experiment to evaluate the feasibility of the proposed system. Two of the subjects have experience in SSVEP based BCI and the others are naïve to BCI. The subjects are undergraduate or postgraduate students of the university. Subjects were provided and signed the consent form and read the information sheet before the experiment. The experiments were approved by the departmental Ethics Committee.

\section{B. Data Acquisition}

SSVEP is found most prominent at the area over the visual cortex $[3,15]$ which is the region of interest (ROI) in this study. 11 electrodes at the occipital region were selected as the signal channels according to the international $10-20$ electrode system. $\mathrm{Cz}$ and $\mathrm{Fz}$ were chosen as the ground and $\rightarrow$ the reference electrode respectively. Fig. 1 shows the channels montage and electrode selection. Subjects were seated on a comfortable chair and wore a 128 channels EEG cap (EASYCAP) in a dim lit room. EEG abrasive skin prepping gel (Nuprep Gel) and EEG conductive gel (ElectroGel) were applied to the electrode sites to eliminate the dead skin and reduce the impedance between scalp and electrodes. The impedance was kept under $5 \mathrm{k} \Omega$ throughout the entire experiment. EEG was recorded by SynAmps ${ }^{2}$ (amplifier, NEUROSCAN) and NeuroScan 4.5 (recording software, NEUROSCAN). EEG was digitized by sampling frequency $2,000 \mathrm{~Hz}$.

\section{Visual stimuli and experiment protocol}

The visual stimuli were four red LEDs (OSRAM $^{\mathrm{TM}}$, LRCP7P which is a surface mount LED being mounted to a substrate $20 \mathrm{~mm} \times 20 \mathrm{~mm}$ ). Four LED substrates were attached at four corners of a rectangular $(25 \mathrm{~cm} \times 25 \mathrm{~cm})$ layout in the centre of a board $(60 \mathrm{~cm} \times 40 \mathrm{~cm})$. Each LED was modulated by a square wave with a distinct frequency generated by a customized circuit based on a microcontroller (MICROCHIP $^{\mathrm{TM}}$, PIC18F46K20). The microcontroller was programmed to generate four square waves of four different frequencies, 12, 13, 14 and $15 \mathrm{~Hz}$. These stimulating frequencies have been widely used in SSVEP based BCI [16]. The duty cycle of the square waves was $50 \%$.

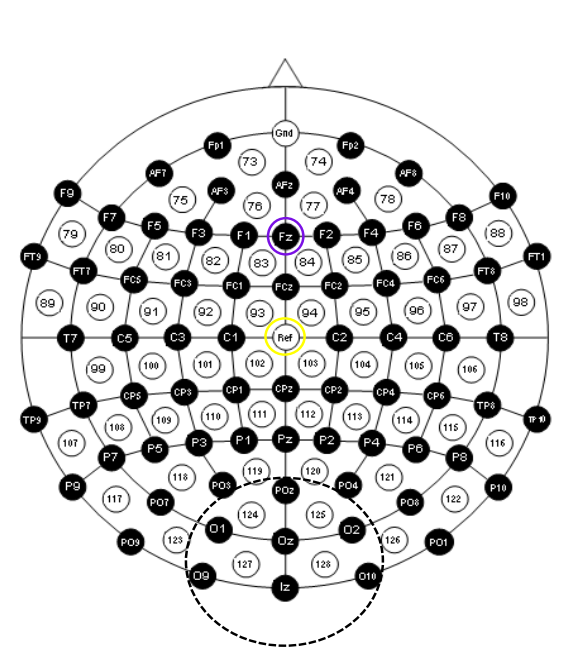

Figure 1: Channels montage. 11 electrodes over occipital region (in the black circle) were selected as EEG recording electrodes. $\mathrm{Cz}$ was the ground electrode in yellow circle and Fz in purple circle was for the reference. The figure of the electrode positions is from www.easycap.de.

During the experiment, the visual stimulator board comprising 4 LEDs was present to the subject at four viewing distances $60 \mathrm{~cm}, 150 \mathrm{~cm}, 250 \mathrm{~cm}$ and $350 \mathrm{~cm}$. In our previous study [17], it was found that the impact of the viewing distance can be compensated by changing the intensities of the visual stimuli. Therefore, the intensities of LEDs presented to the subjects were different at different viewing distance. The intensities of $60 \mathrm{~cm}$ were the lowest and the ones of $350 \mathrm{~cm}$ were the highest in the experiment. For each subject, the experiment contained 4 blocks. Each block corresponded to one viewing distance. Each block contained 4 runs. Each run corresponded to one stimulating frequency and contained 20 trials. Each trial had one attending phase and one resting phase. During the attending phase, 4 LEDs flickered at different frequencies simultaneously for 5 seconds. During the resting phase, 4 LEDs were off. To avoid the visual adaptation, the resting phase lasted for 5 or 6 seconds randomly. Before each run, the subject was instructed to attend to one of four targets for 20 trials. The viewing distances were presented to the subject in a fixed order of $60-150-250-350 \mathrm{~cm}$. The order of attending targets was counterbalanced in 4 blocks (i.e. 16 runs). Each target was attended in different order in each block. There was a short break between two blocks. Subjects were allowed to shift their gaze and head to attend the target but instructed to avoid the movement during the attending phase.

\section{Data pre-processing and off-line analysis}

EEG was band-pass filtered by $1-50 \mathrm{~Hz}$ before being further analyzed. EEG was extracted from 1 second before stimulus onset to 5 seconds after stimulus onset for each trial. Epoch extraction was performed by EEGLAB toolbox. To reduce the computation time of the classification, EEG was down-sampling at $100 \mathrm{~Hz}$.

Fast Fourier Transform (FFT) was used to visualize the SSVEP response at different viewing distances. FFT was also employed to compute SNR which is an important factor 
for SSVEP in the classification. SNR at frequency $f$ is defined by the equation (1) [18],

$S N R_{f}=\frac{2 \times N \times x_{f}}{\sum_{n=1}^{N}\left[x\left(f-n \times f_{\text {res }}\right)+x\left(f+n \times f_{\text {res }}\right)\right]}$

$x_{f}$ represents FFT power at frequency $f$. $f_{\text {res }}$ in equation (1) is the frequency resolution of FFT spectrum. $N$ is the number of neighboring frequencies of $f . N$ was set to 8 in this study. SNR analysis was to inspect the impact of the viewing distance on SSVEP.

EEG was classified into one of 4 classes by CCA. CCA is a statistical multi-variant technique used to investigate the correlation of two sets of variables. CCA transfers two sets of variable $X$ and $Y$ into two canonical variables $U\left(=X^{\mathrm{T}} w_{x}\right)$ and $V\left(=Y^{T} w_{y}\right)$ such that the correlation coefficient $\rho$ between $U$ and $V$ is maximized. The vectors $w_{x}$ and $w_{y}$ can be found by solving the equation (2)[4],

$$
\max _{w_{x}, w_{y}} \rho(U, V)=\frac{E\left[w_{x}^{T} X Y^{T} w_{y}\right]}{\sqrt{E\left[w_{x}^{T} X X^{T} w_{x}\right] E\left[w_{y}^{T} Y Y^{T} w_{y}\right]}}
$$

CCA was first proposed to detect SSVEP by [8]. CCA computes more than one pair of the canonical variables. The correlation coefficient of the first pair canonical variables is the largest and the most important and is used in SSVEP detection in this study. For SSVEP detection in this study, two sets of variables are 11 electrode signals and the reference signal corresponding to one of the of SSVEP, please see $[4,8]$.

\section{RESULTS}

Fig. 2 illustrates the grand average FFT powers of each attended frequency across the subjects at different viewing distances. The dot matrix beside the legend stands for the ANOVA test of FFT powers of 20 trials between the corresponding viewing distance (the row) and one of the other distinct viewing distances (the columns). The diamond, circle and square dots represent the distances $150 \mathrm{~cm}, 250 \mathrm{~cm}$ and $350 \mathrm{~cm}$ respectively. For example, the row beside the legend of $60 \mathrm{~cm}$ stands for ANOVA tests between $60 \mathrm{~cm}$ and $150 \mathrm{~cm}$ (diamond), $250 \mathrm{~cm}$ (circle) and $350 \mathrm{~cm}$ (square) respectively. The unfilled black dots stand for $p$ values of ANOVA tests larger than 0.01 (i.e. not significant). The red filled dots stand for the corresponding $p$ values less than 0.01 (i.e. the significance level is 0.01 ).

In spite of the LED intensities compensated, the grand average of FFT powers still shows significantly different between different viewing distances, especially between $60 \mathrm{~cm}$ and other distances as seen in Fig. 2. Meanwhile SSVEP components at unattended frequencies can be seen in the frequency spectrum. SSVEP response at the attended frequency is lower at the longer viewing distance but SSVEPs of the unattended frequencies are higher at the longer viewing distances compared to the ones at the shorter viewing distances.

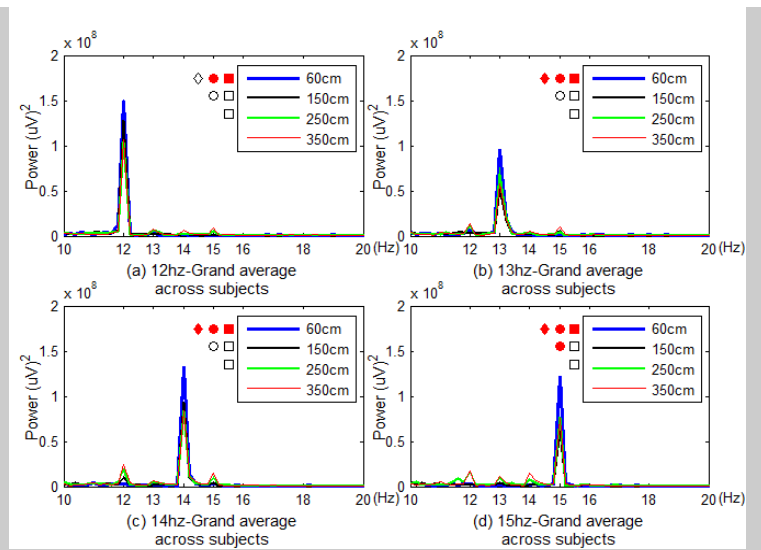

Figure 2: Grand average of FFT power across all subjects of each attended frequency at different viewing distances. The dot matrix beside the legend stands for the ANOVA test of FFT powers of 20 trials between two distinct viewing distances. The diamond, circle and square dots represent the distances $150 \mathrm{~cm}, 250 \mathrm{~cm}$ and $350 \mathrm{~cm}$ respectively. For example, the row beside the legend of $60 \mathrm{~cm}$ stands for ANOVA results between $60 \mathrm{~cm}$ and $150 \mathrm{~cm}$ (diamond), $250 \mathrm{~cm}$ (circle) and $350 \mathrm{~cm}$ (square) respectively. The black unfilled dots stand for the corresponding $p$ values of ANOVA tests $>0.01$ (significance level $=0.01$ ). The red filled dots stand for the corresponding $p$ values $<$ 0.01 .

Fig. 3 is SNRs across 10 subjects of each attended frequency at different viewing distances at 3 seconds after the stimulus onset and their corresponding ANOVA tests. SNRs across the subjects at $60 \mathrm{~cm}$ are the highest among all viewing distances. The blue vertical bars (error bars) indicate the inter-subject variance in SNRs.

Fig. 4 is the mean accuracies of the attended frequency across the subjects at different viewing distances and the corresponding ANOVA tests. EEG epoch time interval of Fig. 4 is 2 seconds and the number of SSVEP harmonics is 1 . Fig. 4 shows inter-subject variance at different viewing distances within the same frequency. Intersubject variance of $12 \mathrm{~Hz}$ is the least prominent while $15 \mathrm{~Hz}$ is the greatest. However, the differences of the mean accuracies at different viewing distances are not significant for all attended frequencies. In our previous study [17], the accuracies at $250 \mathrm{~cm}$ and $350 \mathrm{~cm}$ were very low without intensities compensation.
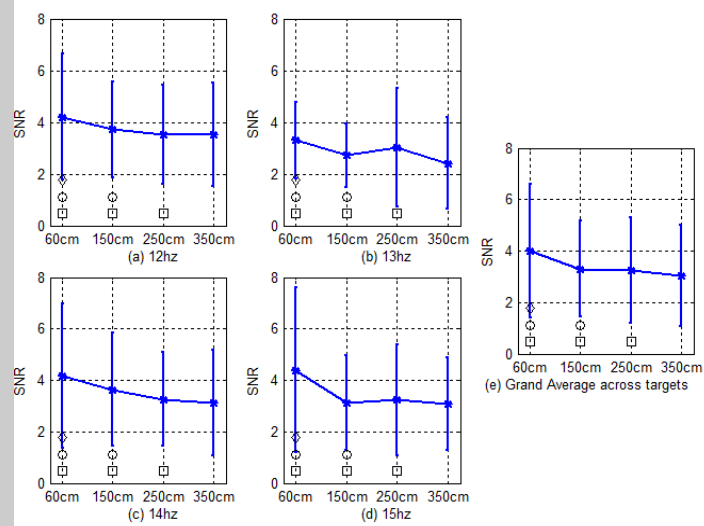

Figure 3: (a)-(d) SNRs of each attended frequency across the subjects at 4 viewing distances. (e) SNRs across the subjects and the attended frequencies corresponding to the same viewing distance. The dots at each viewing distance represent ANOVA tests between SNRs of all subjects at two different viewing distances. The shapes of the dots are the same as Fig. 2. 

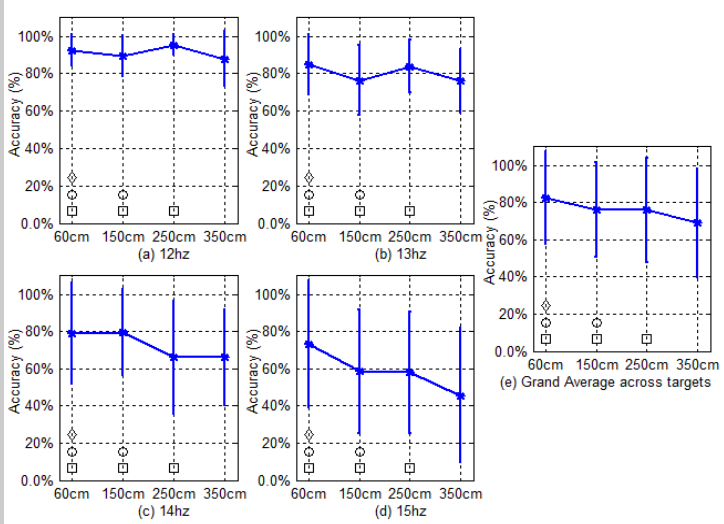

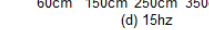

Figure 4: (a)-(d) Mean classification accuracies of the attended frequency across the subjects at different viewing distances and the corresponding ANOVA tests. (e) Mean accuracies across the subjects and the attended frequencies at different viewing distances. The shapes of the dots are the same as Fig. 2.

\section{DISCUSSION}

Even though the intensities of the LEDs were compensated to respond to the change of the viewing distances, the impact of the viewing distance cannot be completely eliminated. This impact can be observed from Fig. 2, half of the ANOVA tests of FFT powers at the different viewing distances which are significant, especially the tests between $60 \mathrm{~cm}$ and other distances for any attended target. However, SNRs of Fig. 3 demonstrate that although SNRs of $60 \mathrm{~cm}$ are higher than $\rightarrow^{\text {the }}$ other viewing distances, the difference is not significant. If the viewing distance of $60 \mathrm{~cm}$ is used as the benchmark, the results demonstrate that SSVEP elicited at longer viewing distances can also be used as input to the BCI system. Furthermore, the mean classification accuracies of all attended targets using 2 seconds of EEG epoch time interval are between $82 \%$ and $68 \%$. These results demonstrate that SSVEP response can be elicited at the longer viewing distances and the detection rate of SSVEP is acceptable. The proposed novel distance adaptable SSVEP BCI is achievable with promising results.

From Fig. 2, it can be seen that SSVEP components of all the stimulating frequencies appear in the FFT power spectrum. Moreover, the components of unattended frequencies become more prominent as the viewing distance increases. This might explain why the classification accuracies at the longer viewing distance are lower than the short viewing distance. The lower classification accuracies might be resulting from the interference of the unattended targets. However, as mentioned in Section II, the viewing distances were presented to the subjects in a fixed order of 60-150-250$350 \mathrm{~cm}$. It cannot be ruled out the poor performance at longer viewing distance is caused by the fatigue. For example, $350 \mathrm{~cm}$ is always the last block in the experiment for all subjects.

The next step of this study is to implement a real time online distance adaptable SSVEP BCI which can detect the user's positions automatically and adjust the intensities of the visual stimuli accordingly. Another direction is to investigate if the combination of the different stimulating frequencies can improve the classification accuracies.

\section{REFERENCES}

[1] J. R. Wolpaw, N. Birbaumer, W. J. Heetderks, D. J. McFarland, P. H Peckham, G. Schalk, et al., "Brain-computer interface technology: A review of the first international meeting," IEEE Transactions on Rehabilitation Engineering, vol. 8, pp. 164-173, Jun 2000.

[2] J. J. Shih, D. J. Krusienski, and J. R. Wolpaw., "Brain-computer interfaces in medicine," in Mayo Clinic Proceedings, 2012, pp. 268 279.

[3] P. Martinez, H. Bakardjian, and A. Cichocki, "Fully online multicommand brain-computer interface with visual neurofeedback using SSVEP paradigm," Computational intelligence and neuroscience, pp. 13-13, 20072007.

[4] G. Bin, X. Gao, Z. Yan, B. Hong, and S. Gao, "An online multichannel SSVEP-based brain-computer interface using a canonical correlation analysis method," Journal of Neural Engineering, vol. 6 , Aug 2009.

[5] I. Volosyak, "SSVEP-based Bremen-BCI interface-boosting information transfer rates," Journal of Neural Engineering, vol. 8, Jun 2011.

[6] X. R. Gao, D. F. Xu, M. Cheng, and S. K. Gao, "A BCI-based environmental controller for the motion-disabled," IEEE Transactions on Neural Systems and Rehabilitation Engineering, vol. 11, pp. 137 140, Jun 2003.

[7] H.-J. Hwang, J.-H. Lim, Y.-J. Jung, H. Choi, S. W. Lee, and C.-H. Im "Development of an SSVEP-based BCI spelling system adopting a QWERTY-style LED keyboard," Journal of Neuroscience Methods, vol. 208, pp. 59-65, Jun 302012.

[8] Z. Lin, C. Zhang, W. Wu, and X. Gao, "Frequency recognition based on canonical correlation analysis for SSVEP-based BCIs," IEEE Transactions on Biomedical Engineering, vol. 53, pp. 2610-2614, Dec 2006.

[9] O. Friman, I. Volosyak, and A. Graeser, "Multiple channel detection of steady-state visual evoked potentials for brain-computer interfaces," IEEE Transactions on Biomedical Engineering, vol. 54, pp. 742-750, Apr 2007.

[10] C. Jia, X. Gao, B. Hong, and S. Gao, "Frequency and Phase Mixed Coding in SSVEP-Based Brain-Computer Interface," IEEE Transactions on Biomedical Engineering, vol. 58, pp. 200-206, Jan 2011.

[11] K.-K. Shyu, P.-L. Lee, Y.-J. Liu, and J.-J. Sie, "Dual-frequency steady-state visual evoked potential for brain computer interface," Neuroscience Letters, vol. 483, pp. 28-31, Oct 82010.

[12] H.-J. Hwang, D. H. Kim, C.-H. Han, and C.-H. Im, "A new dualfrequency stimulation method to increase the number of visual stimuli for multi-class SSVEP-based brain-computer interface (BCI)," Brain Research, vol. 1515, pp. 66-77, Jun 172013.

[13] Y. Zhang, P. Xu, T. Liu, J. Hu, R. Zhang, and D. Yao, "Multiple Frequencies Sequential Coding for SSVEP-Based Brain-Computer Interface," Plos One, vol. 7, Mar 62012.

[14] Y.-P. Lin, Y. Wang, and T.-P. Jung, "Assessing the feasibility of online SSVEP decoding in human walking using a consumer EEG headset," Journal of Neuroengineering and Rehabilitation, vol. 11, Aug 92014

[15] S. T. Morgan, J. C. Hansen, and S. A. Hillyard, "Selective attention to stimulus location modulates the steady-state visual evoked potential," Proceedings of the National Academy of Sciences of the United States of America, vol. 93, pp. 4770-4774, May 141996.

[16] D. Zhu, J. Bieger, G. Garcia Molina, and R. M. Aarts, "A survey of stimulation methods used in SSVEP-based BCIs," Computational intelligence and neuroscience, p. 702357, 2010.

[17] C. Wu and H. Lakany, "The effect of the viewing distance of stimulus on SSVEP response for use in Brain-Computer Interfaces," IEEE International Conference on Systems, Man, and Cybernetics (SMC), pp. 1840-1845, Oct 13-16 2013.

[18] Y. Wang, R. P. Wang, X. R. Gao, B. Hong, and S. K. Gao, "A practical VEP-based brain-computer interface," IEEE Transactions on Neural Systems and Rehabilitation Engineering, vol. 14, pp. 234-239, Jun 2006. 